\title{
A SHARP ONE-SIDED BOUND FOR THE HILBERT TRANSFORM
}

\author{
ADAM OSȨKOWSKI
}

(Communicated by Mark M. Meerschaert)

\begin{abstract}
Let $\mathcal{H}^{\mathbb{T}}, \mathcal{H}^{\mathbb{R}}$ denote the Hilbert transforms on the circle and real line, respectively. The paper contains the proofs of the sharp estimates
\end{abstract}

$$
\left|\left\{\zeta \in \mathbb{T}: \mathcal{H}^{\mathbb{T}} f(\zeta) \geq 1\right\}\right| \leq 2 \pi|| f \|_{1}, \quad f \in L^{1}(\mathbb{T}),
$$

and

$$
\left|\left\{x \in \mathbb{R}: \mathcal{H}^{\mathbb{R}} f(x) \geq 1\right\}\right| \leq\|f\|_{1}, \quad f \in L^{1}(\mathbb{R}) .
$$

A related estimate for orthogonal martingales is also established.

\section{INTRODUCTION}

Our motivation comes from a very basic question about the Hilbert transform $\mathcal{H}^{\mathbb{T}}$ on the unit circle. Recall that this operator is given by the singular integral

$$
\mathcal{H}^{\mathbb{T}} f\left(e^{i \theta}\right)=\frac{1}{2 \pi} \text { p.v. } \int_{-\pi}^{\pi} f(t) \cot \frac{\theta-t}{2} \mathrm{~d} t \quad \text { for } f \in L^{1}(\mathbb{T}) .
$$

A classical result of M. Riesz [10] states that for any $1<p<\infty$ there is a finite universal constant $C_{p}$ such that

$$
\left\|\mathcal{H}^{\mathbb{T}} f\right\|_{p} \leq C_{p}\|f\|_{p}, \quad f \in L^{p}(\mathbb{T}) .
$$

For $p=1$ the above estimate does not hold with any $C_{1}<\infty$, but, as Kolmogorov 8 has shown, there is an absolute $c_{1}<\infty$ such that

$$
(2 \pi)^{-1}\left|\left\{\zeta \in \mathbb{T}:\left|\mathcal{H}^{\mathbb{T}} f(\zeta)\right| \geq 1\right\}\right| \leq c_{1}\|f\|_{1}, \quad f \in L^{1}(\mathbb{T}) .
$$

The optimal values of the constants $C_{p}$ and $c_{1}$ were determined in the seventies: Pichorides [9] and Cole (unpublished; see Gamelin [6]) proved that the best constant in (1.1) equals $\cot \frac{\pi}{2 p^{*}}$, where $p^{*}=\max \{p, p /(p-1)\}$, and Davis [4] showed that the optimal choice for the constant $c_{1}$ in (1.2) is

$$
\left(\frac{1}{\pi} \int_{\mathbb{R}} \frac{\left|\frac{2}{\pi} \log \right| t||}{t^{2}+1} \mathrm{~d} t\right)^{-1}=\frac{1+\frac{1}{3^{2}}+\frac{1}{5^{2}}+\frac{1}{7^{2}}+\ldots}{1-\frac{1}{3^{2}}+\frac{1}{5^{2}}-\frac{1}{7^{2}}+\ldots}=1.347 \ldots
$$

Received by the editors June 27, 2011.

2010 Mathematics Subject Classification. Primary 31B05, 60G44; Secondary 42A50, 42A61.

Key words and phrases. Hilbert transform, martingale, differential subordination, weak type inequality, best constants.

This research was partially supported by MNiSW Grant N N201 397437.

(C)2012 American Mathematical Society Reverts to public domain 28 years from publication 
We turn to the nonperiodic case. Recall that the Hilbert transform $\mathcal{H}^{\mathbb{R}}$ on the real line is defined by

$$
\mathcal{H}^{\mathbb{R}} f(x)=\frac{1}{\pi} \mathrm{p} . \mathrm{v} \cdot \int_{\mathbb{R}} \frac{f(t)}{x-t} \mathrm{~d} t \quad \text { for } f \in L^{1}(\mathbb{R}) .
$$

The above strong- and weak-type inequalities can be extended to analogous statements for $\mathcal{H}^{\mathbb{R}}$, and the optimal constants remain unchanged (see e.g. [10, [12]).

The objective of this paper is to determine the best constant in the one-sided version of the weak type estimate. The result is the following.

Theorem 1.1. We have

$$
\begin{array}{rlrl}
(2 \pi)^{-1}\left|\left\{\zeta \in \mathbb{T}: \mathcal{H}^{\mathbb{T}} f(\zeta) \geq 1\right\}\right| & \leq\|f\|_{1} & & \text { for any } f \in L^{1}(\mathbb{T}), \\
& \left|\left\{x \in \mathbb{R}: \mathcal{H}^{\mathbb{R}} f(x) \geq 1\right\}\right| \leq\|f\|_{1} & \text { for any } f \in L^{1}(\mathbb{R}) .
\end{array}
$$

Both estimates are sharp.

In fact, we shall establish a more general statement in martingale theory. Let $(\Omega, \mathcal{F}, \mathbb{P})$ be a complete probability space, filtered by $\left(\mathcal{F}_{t}\right)_{t \geq 0}$, a nondecreasing family of sub- $\sigma$-algebras of $\mathcal{F}$. Assume further that $\mathcal{F}_{0}$ contains all the events of probability 0 . Let $X=\left(X_{t}\right)_{t>0}, Y=\left(Y_{t}\right)_{t>0}$ be two adapted real martingales with continuous paths and let $[\bar{X}, Y]$ denote their quadratic covariance process (see e.g. Dellacherie and Meyer [5] for details). We say that the processes $X$ and $Y$ are orthogonal if $[X, Y]$ is constant almost surely. Following Bañuelos and Wang [1] and Wang [11, we say that $Y$ is differentially subordinate to $X$ if the process $\left([X, X]_{t}-[Y, Y]_{t}\right)_{t \geq 0}$ is nondecreasing and nonnegative as a function of $t$.

Bañuelos and Wang [1, 22 proved the following versions of (1.1) and (1.2) (see also Choi [3] and Janakiraman [7] for related results). Here and below, we use the notation $\|X\|_{p}=\sup _{t \geq 0}\left\|X_{t}\right\|_{p}$ for $1 \leq p<\infty$.

Theorem 1.2. Assume that $X, Y$ are orthogonal martingales such that $Y$ is differentially subordinate to $X$. Then

$$
\|Y\|_{p} \leq \cot \frac{\pi}{2 p^{*}}\|X\|_{p}
$$

for $1<p<\infty$ and

$$
\mathbb{P}\left(\sup _{t \geq 0}\left|Y_{t}\right| \geq 1\right) \leq\left(\frac{1}{\pi} \int_{\mathbb{R}} \frac{\left|\frac{2}{\pi} \log \right| t||}{t^{2}+1} d t\right)^{-1}\|X\|_{1} .
$$

Both estimates are sharp.

We shall establish the following probabilistic counterpart of Theorem 1.1.

Theorem 1.3. Assume that $X, Y$ are orthogonal martingales such that $Y$ is differentially subordinate to $X$ and $Y_{0}=0$. Then

$$
\mathbb{P}\left(\sup _{t \geq 0} Y_{t} \geq 1\right) \leq\|X\|_{1}
$$

and the inequality is sharp.

A few words about the organization of the paper. The proofs of (1.3) and (1.4) rest on the existence of a certain special superharmonic function. The method is explained in Section 2, and the function is constructed in Section 3. In the final part of the paper we show that the one-sided estimates do not hold with any constant smaller than 1. 


\section{Proofs of (1.3) AND (1.4)}

The central role in the paper is played by the following special function on $\mathbb{R}^{2}$.

Theorem 2.1. There is a continuous function $U: \mathbb{R}^{2} \rightarrow \mathbb{R}$ which satisfies the following properties:

(i) For any $x, y \in \mathbb{R}$ we have $U(x, y) \geq 1_{\{y \leq 0\}}-|x|$.

(ii) For any $x \in \mathbb{R}$ we have $U(x, 1) \leq 0$.

(iii) For any $y \in \mathbb{R}$, the function $U(\cdot, y): x \mapsto U(x, y)$ is concave on $\mathbb{R}$.

(iv) $U$ is superharmonic.

This theorem will be shown in the next section. Now let us see how it leads to the announced estimates.

Proof of (1.4). Consider a $C^{\infty}$ radial function $g: \mathbb{R}^{2} \rightarrow[0, \infty)$, supported on the ball of center $(0,0)$ and radius 1 , satisfying $\int_{\mathbb{R}^{2}} g=1$. For any $\delta>0$, define $U^{\delta}: \mathbb{R}^{2} \rightarrow \mathbb{R}$ by the convolution

$$
U^{\delta}(x, y)=\int_{\mathbb{R}^{2}} U(x+\delta r, y+\delta s) g(r, s) \mathrm{d} r \mathrm{~d} s .
$$

Clearly, the function $U^{\delta}$ is of class $C^{\infty}$ and inherits the concavity along the horizontal lines as well as the superharmonicity property. In addition, we have the majorization $U \geq U^{\delta}$, since $U$ is superharmonic and $g$ is radial. Consequently,

$$
U^{\delta}(x, 1) \leq 0 \quad \text { for any } x \in \mathbb{R} .
$$

Next, observe that by (i),

$$
\begin{aligned}
U^{\delta}(x, y) & \geq \int_{\mathbb{R}^{2}} 1_{\{y+\delta s \leq 0\}} g(r, s) \mathrm{d} r \mathrm{~d} s-\int_{\mathbb{R}^{2}}|x+\delta r| g(r, s) \mathrm{d} r \mathrm{~d} s \\
& \geq 1_{\{y \leq-\delta\}}-(|x|+\delta) .
\end{aligned}
$$

Let $X, Y$ be martingales as in the statement. Using localization, we may assume that $X, Y$ are bounded; this will guarantee the integrability of all the random variables appearing below. Fix $\varepsilon>0$ and introduce the stopping time $\tau=\inf \{t \geq$ $\left.0: Y_{t} \geq 1+\varepsilon\right\}$. An application of Itô's formula gives

$$
U^{\delta}\left(X_{\tau \wedge t}, 1-Y_{\tau \wedge t}\right)=U^{\delta}\left(X_{0}, 1-Y_{0}\right)+I_{1}+I_{2} / 2
$$

where

$$
\begin{aligned}
I_{1}= & \int_{0+}^{\tau \wedge t} U_{x}^{\delta}\left(X_{s}, 1-Y_{s}\right) \mathrm{d} X_{s}+\int_{0+}^{\tau \wedge t} U_{y}^{\delta}\left(X_{s}, 1-Y_{s}\right) \mathrm{d} Y_{s} \\
I_{2}= & \int_{0+}^{\tau \wedge t} U_{x x}^{\delta}\left(X_{s}, 1-Y_{s}\right) \mathrm{d}[X, X]_{s} \\
& +2 \int_{0+}^{\tau \wedge t} U_{x y}^{\delta}\left(X_{s}, 1-Y_{s}\right) \mathrm{d}[X, Y]_{s}+\int_{0+}^{\tau \wedge t} U_{y y}^{\delta}\left(X_{s}, 1-Y_{s}\right) \mathrm{d}[Y, Y]_{s} .
\end{aligned}
$$

Observe that $U^{\delta}\left(X_{0}, 1-Y_{0}\right)=U^{\delta}\left(X_{0}, 1\right) \leq 0$ by virtue of (2.1). Next, we have $\mathbb{E} I_{1}=0$, since both stochastic integrals in $\bar{I}_{1}$ are martingales. Using the orthogonality of $X$ and $Y$, we see that the middle term in $I_{2}$ vanishes. Combining this with the inequality $U_{x x}^{\delta} \leq 0$ and the differential subordination of $Y$ to $X$, we obtain

$$
I_{2} \leq \int_{0+}^{\tau \wedge t} U_{x x}^{\delta}\left(X_{s}, 1-Y_{s}\right) \mathrm{d}[Y, Y]_{s}+\int_{0+}^{\tau \wedge t} U_{y y}^{\delta}\left(X_{s}, 1-Y_{s}\right) \mathrm{d}[Y, Y]_{s},
$$


which is nonpositive since $U^{\delta}$ is superharmonic. Plugging all these facts into (2.3) gives $\mathbb{E} U^{\delta}\left(X_{\tau \wedge t}, 1-Y_{\tau \wedge t}\right) \leq 0$, and hence, by (2.2),

$$
\mathbb{P}\left(Y_{\tau \wedge t} \geq 1+\delta\right) \leq \mathbb{E}\left(\left|X_{\tau \wedge t}\right|+\delta\right) .
$$

Letting $\delta \rightarrow 0$, we get $\mathbb{P}\left(Y_{\tau \wedge t}>1\right) \leq \mathbb{E}\left|X_{\tau \wedge t}\right| \leq\|X\|_{1}$. Therefore,

$$
\mathbb{P}\left(\sup _{t \geq 0} Y_{t} \geq 1+2 \varepsilon\right) \leq \lim _{t \rightarrow \infty} \mathbb{P}\left(\left|Y_{\tau \wedge t}\right|>1\right) \leq\|X\|_{1}
$$

It suffices to apply this bound to a new pair $((1+2 \varepsilon) X,(1+2 \varepsilon) Y)$ (for which the orthogonality and differential subordination hold) and let $\varepsilon \rightarrow 0$.

Proof of (1.3) in the periodic case. This is standard. Let $B$ be a planar Brownian motion starting from $0 \in \mathbb{C}$ and let $\tau=\inf \left\{t \geq 0:\left|B_{t}\right|=1\right\}$. Let $u, v$ be the harmonic extensions (by Poisson integrals) of $f$ and $\mathcal{H}^{\mathbb{T}} f$ to the unit disc. Then $u, v$ satisfy Cauchy-Riemann equations, and we have $v(0)=0$. Thus the martingales $X=\left(u\left(B_{\tau \wedge t}\right)\right)_{t \geq 0}, Y=\left(v\left(B_{\tau \wedge t}\right)\right)_{t \geq 0}$ are orthogonal, $Y$ is differentially subordinate to $X$ and $Y_{0}=0$. To verify these conditions, use the identities

$$
[X, X]_{t}=|u(0)|^{2}+\int_{0+}^{\tau \wedge t}\left|\nabla u\left(B_{s}\right)\right|^{2} \mathrm{~d} s, \quad[Y, Y]_{t}=\int_{0+}^{\tau \wedge t}\left|\nabla v\left(B_{s}\right)\right|^{2} \mathrm{~d} s
$$

and

$$
[X, Y]_{t}=\int_{0+}^{\tau \wedge t} \nabla u\left(B_{s}\right) \cdot \nabla v\left(B_{s}\right) \mathrm{d} s .
$$

Consequently, since $B_{\tau}$ is uniformly distributed on the unit circle, we obtain

$$
(2 \pi)^{-1}\left|\left\{\zeta \in \mathbb{T}: \mathcal{H}^{\mathbb{T}} f(\zeta) \geq 1\right\}\right| \leq \mathbb{P}\left(\sup _{t} Y_{t} \geq 1\right) \leq\|X\|_{1}=\|u\|_{1} .
$$

Proof of (1.3) in the nonperiodic case. To deduce the weak-type estimate for the Hilbert transform on the line, we use a standard argument known as "blowing up the circle", which is due to Zygmund ([12, Chapter XVI, Theorem 3.8). Let $f$ be an integrable function on $\mathbb{R}$. For a given positive integer $n$ and $x \in \mathbb{R}$, put

$$
g_{n}(x)=\frac{1}{2 \pi n} \mathrm{p} \cdot \mathrm{v} \cdot \int_{-\pi n}^{\pi n} f(t) \cot \frac{x-t}{2 n} \mathrm{~d} t .
$$

As shown in [12, we have $g_{n} \rightarrow \mathcal{H}^{\mathbb{R}} f$ almost everywhere as $n \rightarrow \infty$. On the other hand, the function

$$
x \mapsto g_{n}(n x)=\frac{1}{2 \pi} \mathrm{p} . \mathrm{v} \cdot \int_{-\pi}^{\pi} f(n t) \cot \frac{x-t}{2} \mathrm{~d} t
$$

is the periodic Hilbert transform of the function $f_{n}: x \mapsto f(n x),|x| \leq \pi$, so

$$
\begin{aligned}
\left|\left\{x \in(-\pi n, \pi n]: g_{n}(x) \geq 1\right\}\right| & =n\left|\left\{|x| \in(-\pi, \pi]: \mathcal{H}^{\mathbb{T}} f_{n}(x) \geq 1\right\}\right| \\
& \leq n \int_{-\pi}^{\pi}\left|f_{n}(x)\right| \mathrm{d} x=\int_{-\pi n}^{\pi n}|f(x)| \mathrm{d} x \leq\|f\|_{1} .
\end{aligned}
$$

Now let $n \rightarrow \infty$ to obtain $\left|\left\{x \in \mathbb{R}: \mathcal{H}^{\mathbb{R}} f(x)>1\right\}\right| \leq\|f\|_{1}$. To get the nonstrict inequality on the left, pick $\varepsilon>0$ and apply the above estimate to $f /(1-\varepsilon)$. Then

$$
\left|\left\{x \in \mathbb{R}: \mathcal{H}^{\mathbb{R}} f(x) \geq 1\right\}\right| \leq\left|\left\{x \in \mathbb{R}: \mathcal{H}^{\mathbb{R}} f(x)>1-\varepsilon\right\}\right| \leq \frac{1}{1-\varepsilon}\|f\|_{1},
$$

and it remains to let $\varepsilon \rightarrow 0$. 


\section{A special Function - Proof of Theorem 2.1}

Throughout, $H$ will denote the upper half-plane $\mathbb{R} \times(0, \infty)$. Introduce the function $\mathcal{U}: H \rightarrow \mathbb{R}$ given by the Poisson integral

$$
\mathcal{U}(\alpha, \beta)=\frac{1}{\pi} \int_{0}^{\infty} \frac{\beta\left(1-\frac{1}{2}\left|\sqrt{t}-\sqrt{t^{-1}}\right|\right)}{(\alpha-t)^{2}+\beta^{2}} \mathrm{~d} t .
$$

The function $\mathcal{U}$ is harmonic on $H$ and satisfies, for $\alpha \neq 0$,

$$
\lim _{\beta \downarrow 0} \mathcal{U}(\alpha, \beta)=\left(1-\frac{1}{2}\left|\sqrt{\alpha}-\sqrt{\alpha^{-1}}\right|\right) 1_{\{\alpha>0\}} .
$$

Let $K$ be the conformal mapping of $H$ onto $H \backslash\{a i: a \geq 1\}$, defined by

$$
K(z)=\frac{1}{2}\left(\sqrt{z}-\frac{1}{\sqrt{z}}\right)
$$

and let $L$ stand for its inverse. We easily derive that

$$
L(z)=2 z^{2}+1+2 z \sqrt{z^{2}+1} .
$$

Here and below we use the following branch of the complex square root: if $z=r e^{i \theta}$ for some $r \geq 0$ and $\theta \in(-\pi, \pi]$, then $\sqrt{z}=\sqrt{r} e^{i \theta / 2}$.

Now we are ready to introduce the special function. First we define it on the set $H \backslash\{a i: a \geq 1\}$ by $U(x, y)=\mathcal{U}(L(x, y))$. Using (3.1), we see that $U$ can be extended to a continuous function on $\mathbb{R}^{2}$ by putting $U(x, y)=1-|x|$ on $\mathbb{R} \times(-\infty, 0]$ and $U(0, y)=0$ for $y \geq 1$.

Lemma 3.1. The function $U$ enjoys the following properties:

(i) $U$ is harmonic on $H \backslash\{a i: a \geq 1\}$.

(ii) The function $(x, y) \mapsto U(x, y)+|x|$ is bounded on $\mathbb{R}^{2}$.

(iii) $U$ satisfies the symmetry condition $U(x, y)=U(-x, y)$ for all $x, y$.

Proof. (i) This is obvious: $\mathcal{U}$ is harmonic on $H$, so the function $U$ is a real part of an analytic function on $H \backslash\{a i: a \geq 1\}$.

(ii) Of course, it suffices to establish the boundedness on $H$. Introduce the function $A: H \rightarrow \mathbb{R}$ by

$$
A(\alpha, \beta)=\frac{1}{2 \pi} \int_{0}^{\infty} \frac{\beta\left(\sqrt{t}-\sqrt{t^{-1}}\right)}{(\alpha-t)^{2}+\beta^{2}} \mathrm{~d} t .
$$

It is not difficult to prove, using the residuum calculus, that

$$
A(\alpha, \beta)=\frac{1}{\pi} \int_{\mathbb{R}} \frac{\beta\left(s^{2}-1\right)}{\left(\alpha-s^{2}\right)^{2}+\beta^{2}} \mathrm{~d} s=\operatorname{Re} K(\alpha, \beta)
$$

( $K$ is defined by (3.2) ) and hence $A(L(x, y))=x$. Now, when $x, y>0$, then $|L(x, y)| \geq 1$ and $|U(x, y)+| x||=|\mathcal{U}(L(x, y))+A(L(x, y))|$; but, for $\alpha^{2}+\beta^{2} \geq 1$,

$$
|U(\alpha, \beta)+A(\alpha, \beta)| \leq \frac{1}{\pi} \int_{0}^{\infty} \frac{\beta}{(\alpha-t)^{2}+\beta^{2}} \mathrm{~d} t+\frac{1}{\pi} \int_{0}^{1} \frac{\beta\left(\sqrt{t^{-1}}-\sqrt{t}\right)}{(\alpha-t)^{2}+\beta^{2}} \mathrm{~d} t \leq C
$$

for some absolute $C$. Similarly, if $x<0$ and $y>0$, then $|L(x, y)| \leq 1, \mid U(x, y)+$ $|x||=| \mathcal{U}(L(x, y))-A(L(x, y)) \mid$ and, for $\alpha^{2}+\beta^{2} \leq 1$ and some universal $C$,

$$
|U(\alpha, \beta)-A(\alpha, \beta)| \leq \frac{1}{\pi} \int_{0}^{\infty} \frac{\beta}{(\alpha-t)^{2}+\beta^{2}} \mathrm{~d} t+\frac{1}{\pi} \int_{1}^{\infty} \frac{\beta\left(\sqrt{t}-\sqrt{t^{-1}}\right)}{(\alpha-t)^{2}+\beta^{2}} \mathrm{~d} t \leq C .
$$


(iii) The function $S(x, y)=U(x, y)-U(-x, y)$ is continuous on $\mathbb{R}^{2}$, harmonic on $H \backslash\{a i: a \geq 1\}$ and $S=0$ on $\{(x, y): y \leq 0$ or $x=0\}$. Furthermore, $S$ is bounded, in view of the previous part. Thus $S \equiv 0$.

To study the further properties of $U$, we shall need the following family of auxiliary functions. For $b>1$, let $f_{b}:[1, \infty) \rightarrow \mathbb{R}$ be given by

$$
f_{b}=1_{[1, b]}-\frac{b-1}{2} 1_{(b, \infty)} .
$$

Next, let $\Phi_{b}:[0,1] \rightarrow \mathbb{R}$ be defined by the formula

$$
\begin{aligned}
\Phi_{b}(a) & =2 a \sqrt{1-a^{2}} \int_{1}^{\infty} \frac{f_{b}(t)}{t^{2}-2\left(1-2 a^{2}\right) t+1} \mathrm{~d} t \\
& =\frac{b+1}{2} \arctan \frac{b+2 a^{2}-1}{2 a \sqrt{1-a^{2}}}-\arctan \frac{a}{\sqrt{1-a^{2}}}-\frac{(b-1) \pi}{4} .
\end{aligned}
$$

Lemma 3.2. (i) For any $b>1$ the function $\Phi_{b}$ is convex.

(ii) The function $U(0, \cdot)$ is convex on $[0, \infty)$.

Proof. (i) A bit lengthy computation yields

$$
\Phi_{b}^{\prime \prime}(a)=\frac{2 b(b-1) a}{\sqrt{1-a^{2}}\left(b^{2}-2 b\left(1-2 a^{2}\right)+1\right)}+\frac{16 a b^{2}(b-1) \sqrt{1-a^{2}}}{\left(b^{2}-2 b\left(1-2 a^{2}\right)+1\right)^{2}} \geq 0 .
$$

(ii) When $a \in(0,1)$, then $L(a i)=1-2 a^{2}+2 a i \sqrt{1-a^{2}}$ belongs to the unit circle, and hence, using the substitution $t:=1 / t$, we derive that

$$
U(0, a)=\frac{1}{\pi} \int_{1}^{\infty} \frac{2 a \sqrt{1-a^{2}}\left(2-\sqrt{t}+\sqrt{t^{-1}}\right)}{t^{2}-2\left(1-2 a^{2}\right) t+1} \mathrm{~d} t .
$$

However, we have the identity

$$
2-\sqrt{t}+\sqrt{t^{-1}}=\int_{1}^{\infty} f_{b}(t) \frac{\mathrm{d} b}{b^{3 / 2}}, \quad t \geq 1 .
$$

Consequently, applying Fubini's theorem, we obtain that

$$
U(0, a)=\frac{1}{\pi} \int_{1}^{\infty} \Phi_{b}(a) \frac{\mathrm{d} b}{b^{3 / 2}}
$$

and by the previous part, $U(0, \cdot)$ is convex on $[0,1]$. Obviously, this function is also convex on $[1, \infty)$, and hence we will be done if we prove that $\lim _{a \uparrow 1} U_{y}(0, a)=0$. To do this, we differentiate both sides of (3.3) with respect to $a$ and obtain

$$
\begin{aligned}
U_{y}(0, a)= & \frac{2}{\pi} \sqrt{1-a^{2}} \int_{1}^{\infty} \frac{2-\sqrt{t}+\sqrt{t^{-1}}}{t^{2}-2\left(1-2 a^{2}\right) t+1} \mathrm{~d} t \\
& -\frac{2 a \sqrt{1-a^{2}}}{\pi} \int_{1}^{\infty} \frac{\left(2-\sqrt{t}+\sqrt{t^{-1}}\right) \cdot 8 a t}{\left(t^{2}-2\left(1-2 a^{2}\right) t+1\right)^{2}} \mathrm{~d} t \\
& -\frac{2 a^{2}}{\pi \sqrt{1-a^{2}}} \int_{1}^{\infty} \frac{2-\sqrt{t}+\sqrt{t^{-1}}}{t^{2}-2\left(1-2 a^{2}\right) t+1} \mathrm{~d} t
\end{aligned}
$$

Obviously, the first two summands on the right vanish as $a \uparrow 1$. To deal with the third one, we use the integration by parts to get

$$
\int_{1}^{\infty} \frac{2-\sqrt{t}+\sqrt{t^{-1}}}{t^{2}+2 t+1} \mathrm{~d} t=0
$$


and hence

$$
\begin{aligned}
\int_{1}^{\infty} \frac{2-\sqrt{t}+\sqrt{t^{-1}}}{t^{2}-2\left(1-2 a^{2}\right) t+1} \mathrm{~d} t & =\int_{1}^{\infty} \frac{2-\sqrt{t}+\sqrt{t^{-1}}}{t^{2}-2\left(1-2 a^{2}\right) t+1} \mathrm{~d} t-\int_{1}^{\infty} \frac{2-\sqrt{t}+\sqrt{t^{-1}}}{t^{2}+2 t+1} \mathrm{~d} t \\
& =4\left(1-a^{2}\right) \int_{1}^{\infty} \frac{\left(2-\sqrt{t}+\sqrt{t^{-1}}\right) t}{\left(t^{2}-2\left(1-2 a^{2}\right) t+1\right)\left(t^{2}+2 t+1\right)} \mathrm{d} t
\end{aligned}
$$

Therefore the third term in (3.4) tends to 0 as $a \uparrow 1$ and the proof is complete.

Lemma 3.3. We have $U_{x x} \leq 0$ on $H \backslash\{a i: a \geq 1\}$.

Proof. By Lemma 3.1(i), the claim is equivalent to saying that $U_{y y} \geq 0$ on $H \backslash$ $\{a i: a \geq 1\}$. By the symmetry of $U$, it suffices to prove that $U_{y y}(x, y) \geq 0$ for $x, y>0$. Using the Schwarz reflection principle, we see that the continuous function $V:[0, \infty) \times \mathbb{R} \rightarrow \mathbb{R}$, given by

$$
V(x, y)= \begin{cases}U(x, y) & \text { if } y \geq 0 \\ -U(x,-y)-2 x+2 & \text { if } y<0\end{cases}
$$

is harmonic; furthermore, $(x, y) \mapsto V(x, y)+x$ is bounded, in view of Lemma 3.1(ii). Fix $h>0$ and consider a continuous function $W$ on $[0, \infty) \times[0, \infty)$, defined by

$$
W(x, y)=2 V(x, y)-V(x, y-h)-V(x, y+h) .
$$

This function is bounded, harmonic and nonpositive at the boundary of its domain, as we shall now prove. Indeed, $W(x, 0)=2-2 x-(2-2 x)=0$. If $y \geq h$, then

$$
W(0, y)=2 U(0, y)-U(0, y-h)-U(0, y+h) \leq 0,
$$

by Lemma 3.2 (ii). Finally, for $0<y<h$,

$$
\begin{aligned}
V(0, y+h)+V(0, y-h) & =U(0, y+h)+2-U(0, h-y) \\
& =U(0, y+h)+2 U(0,0)-U(0, h-y) \\
& \geq 2 U(0, y)=2 V(0, y),
\end{aligned}
$$

again by Lemma 3.2(ii), because both $y$ and $h-y$ lie between 0 and $y+h$. Consequently, $W \leq 0$, and the claim follows, since $h>0$ was arbitrary.

Proof of Theorem 2.1. (i) We may assume that $x, y>0$. Combining Lemma 3.1(ii) with Lemma 3.3 we get that $U_{x} \geq-1$ on $(0, \infty) \times(0, \infty)$. Hence it suffices to prove that $U(0, y) \geq 0$ for $y>0$, but this follows directly from Lemma 3.2 (ii).

(ii) By the symmetry and harmonicity of $U$ on the strip $\mathbb{R} \times(0,1)$, we have $U_{x}(0, y)=0$ for $y \in(0,1)$. Consequently, by Lemma 3.3. for such a fixed $y$ the function $U(\cdot, y)$ is nonincreasing on $[0, \infty)$. By continuity, this is also true for $y=1$, and hence $U(x, 1)=U(|x|, 1) \leq U(0,1)=0$.

(iii) By Lemma 3.3, $U$ is concave along the line $\mathbb{R} \times\{y\}$ when $y \leq 1$. To deal with $y>1$, it suffices to prove that $U(x, y) \leq 0$ for $x>0$ (again due to Lemma 3.3). However, by Lemma 3.3. for any fixed $x$ the function $U(x, \cdot)$ is convex on $[0, \infty)$. Thus, it is nonincreasing, since otherwise Lemma 3.1(ii) would be violated, and hence, for $y>1, U(x, y) \leq U(x, 1) \leq 0$, as we have just proved above.

(iv) The function $U$ is harmonic on $\mathbb{R}^{2} \backslash\left(S_{1} \cup S_{2} \cup S_{3}\right)$, where $S_{1}=\mathbb{R} \times\{0\}$, $S_{2}=\{a i: a<0\}$ and $S_{3}=\{a i: a \geq 1\}$, and thus all we need is to verify the mean-value inequality for the points from $S_{1} \cup S_{2} \cup S_{3}$. This property is clear on 
$S_{1} \cup S_{2}$, because $U(x, y) \leq 1-|x|$ on $\mathbb{R}^{2}$ (we have shown above that $U(x, \cdot)$ is nonincreasing on $[0, \infty))$. To deal with $S_{3}$, pick $a>1$ and note that for $r<a-1$,

$$
\frac{1}{|B((0, a), r)|} \int_{B((0, a), r)} U(x, y) \mathrm{d} x \mathrm{~d} y \leq 0=U(0, a) .
$$

Thus the claim follows from the continuity of $U$.

\section{Sharpness}

Clearly, it suffices to prove the optimality of the constant 1 in the estimate for $\mathcal{H}^{\mathbb{R}}$. Let $D$ denote the unit disc of $\mathbb{C}$ and consider a conformal mapping $M(z)=$ $-(1-z)^{2} /(4 z)$ of $D \cap H$ onto $H$. Let $N$ stand for the inverse of $M$. We easily compute that $N$ maps $[0,1]$ onto the half-circle $\left\{e^{i \theta}: 0 \leq \theta \leq \pi\right\}$ and the set $\mathbb{R} \backslash[0,1]$ onto $(-1,1)$. More precisely, when $x \in[0,1]$, we have

$$
N(x)=e^{i \theta}, \quad \text { where } \theta \text { is determined by } x=\sin ^{2}(\theta / 2),
$$

and

$$
N(x)= \begin{cases}-2 x+1+2 \sqrt{x^{2}-x} & \text { if } x>1, \\ -2 x+1-2 \sqrt{x^{2}-x} & \text { if } x<0 .\end{cases}
$$

Let $\alpha \in(0,1)$ be a fixed number and let $F$ be another conformal mapping, which maps $D$ onto $H \backslash\{a i: a \geq 1\}$ and satisfies $F(0)=\alpha i$. We may and do assume that $F$ is bounded on the interval $[-1,1]$, composing $F$ with the rotation $z \mapsto \zeta z$ if necessary (for some appropriate $\zeta \in \mathbb{T}$ ). For any positive integer $n$, consider the function $f_{n}: \mathbb{R} \rightarrow \mathbb{R}$ given by $f_{n}(x)=-\operatorname{Re} F\left((N(x))^{2 n}\right)$. Since $F \circ N^{2 n}$ is conformal and $F\left((N(z))^{2 n}\right) \rightarrow \alpha i$ as $z \rightarrow \infty$, we have $\mathcal{H}^{\mathbb{R}} f_{n}(x)=\alpha-\operatorname{Im} F\left((N(x))^{2 n}\right)$. Next,

$$
\int_{\mathbb{R}}\left|f_{n}\right|=\int_{[0,1]}\left|f_{n}\right|+\int_{\mathbb{R} \backslash[0,1]}\left|f_{n}\right| .
$$

Using the above expressions for $N(x)$, we get

$$
\begin{aligned}
\int_{[0,1]}\left|f_{n}\right| & =\int_{[0,1]}\left|\operatorname{Re} F\left((N(x))^{2 n}\right)\right| \mathrm{d} x \\
& =\int_{0}^{\pi}\left|\operatorname{Re} F\left(e^{2 n i \theta}\right)\right| \sin \theta \mathrm{d} \theta \\
& =\int_{0}^{2 n \pi}\left|\operatorname{Re} F\left(e^{i \theta}\right)\right| \sin \left(\frac{\theta}{2 n}\right) \frac{\mathrm{d} \theta}{2 n} \\
& =\int_{0}^{2 \pi}\left|\operatorname{Re} F\left(e^{i \theta}\right)\right| \cdot \frac{1}{2 n} \sum_{k=0}^{n-1} \sin \left(\frac{k \pi}{n}+\frac{\theta}{2 n}\right) \mathrm{d} \theta \\
& =\int_{0}^{2 \pi}\left|\operatorname{Re} F\left(e^{i \theta}\right)\right| \cdot\left[\frac{1+\cos (\pi / n)}{2 n \sin (\pi / n)} \cos \left(\frac{\theta}{2 n}\right)+\sin \left(\frac{\theta}{2 n}\right)\right] \mathrm{d} \theta .
\end{aligned}
$$

When $n \rightarrow \infty$, the expression in the square brackets converges to $1 / \pi$ uniformly on $[0,2 \pi]$, and hence

$$
\lim _{n \rightarrow \infty} \int_{[0,1]}\left|f_{n}\right|=\frac{1}{\pi} \int_{0}^{2 \pi}\left|\operatorname{Re} F\left(e^{i \theta}\right)\right| \mathrm{d} \theta
$$


Next, for any $b>0$, the function $N$ takes values in a closed subinterval of $[0,1)$ when restricted to $(-\infty,-b] \cup[1+b, \infty)$. Consequently, the sequence $N^{2 n}$ converges uniformly to 0 on this set. Furthermore, $\left|f_{n}\right|$ is bounded on $(-b, 0) \cup(1,1+b)$, since $F$ is bounded on $[-1,1]$. This implies $\int_{\mathbb{R} \backslash[0,1]}\left|f_{n}\right| \rightarrow 0$, and hence

$$
\lim _{n \rightarrow \infty}\left\|f_{n}\right\|_{1}=\frac{1}{\pi} \int_{0}^{2 \pi}\left|\operatorname{Re} F\left(e^{i \theta}\right)\right| \mathrm{d} \theta .
$$

A similar calculation shows that

$$
\begin{aligned}
\left|\left\{x \in \mathbb{R}: \mathcal{H}^{\mathbb{R}} f_{n}(x) \geq \alpha\right\}\right| & =\left|\left\{x \in \mathbb{R}: \alpha-\operatorname{Im} F\left((N(x))^{2 n}\right) \geq \alpha\right\}\right| \\
& \geq\left|\left\{x \in[0,1]: \alpha-\operatorname{Im} F\left((N(x))^{2 n}\right) \geq \alpha\right\}\right| \\
& =\int_{\left\{\theta \in[0, \pi]: \operatorname{Im} F\left(e^{2 n i \theta}\right) \leq 0\right\}} \sin \theta \mathrm{d} \theta \\
& \rightarrow \frac{1}{\pi}\left|\left\{\theta \in[0,2 \pi]: \operatorname{Im} F\left(e^{i \theta}\right) \leq 0\right\}\right| .
\end{aligned}
$$

Therefore, since $U \circ F$ is harmonic on $D$ (due to Lemma 3.1(i)), we may write

$$
\begin{aligned}
\mid\{x \in & \left.\mathbb{R}: \mathcal{H}^{\mathbb{R}} f_{n}(x) \geq \alpha\right\}|-| \mid f_{n} \|_{1} \\
& \rightarrow \frac{1}{\pi}\left(\left|\left\{\theta \in[0,2 \pi]: \operatorname{Im} F\left(e^{i \theta}\right) \leq 0\right\}\right|-\int_{0}^{2 \pi}\left|\operatorname{Re} F\left(e^{i \theta}\right)\right| \mathrm{d} \theta\right) \\
& =\frac{1}{\pi} \int_{0}^{2 \pi} U\left(F\left(e^{i \theta}\right)\right) \mathrm{d} \theta=2 U(F(0))=2 U(0, \alpha)>0 .
\end{aligned}
$$

In other words, replacing $f_{n}$ by $f_{n} / \alpha$, we see that there is a function $f$ on $\mathbb{R}$ satisfying $\left|\left\{x \in \mathbb{R}: \mathcal{H}^{\mathbb{R}} f(x) \geq 1\right\}\right| /\|f\|_{1} \geq \alpha$. Letting $\alpha \rightarrow 1$, we get the desired sharpness.

\section{REFERENCES}

[1] R. Bañuelos and G. Wang, Sharp inequalities for martingales with applications to the Beurling-Ahlfors and Riesz transforms, Duke Math. J. 80 (1995), no. 3, 575-600. MR.1370109 (96k:60108)

[2] R. Bañuelos and G. Wang, Davis's inequality for orthogonal martingales under differential subordination, Michigan Math. J. 47 (2000), 109-124. MR1755259 (2001g:60100)

[3] C. Choi, A weak-type inequality for differentially subordinate harmonic functions, Trans. Amer. Math. Soc. 350 (1998), 2687-2696. MR.1617340 (99e:31006)

[4] B. Davis, On the weak type $(1,1)$ inequality for conjugate functions, Proc. Amer. Math. Soc. 44 (1974), 307-311. MR0348381 (50:879)

[5] C. Dellacherie and P. A. Meyer, Probabilities and potential. B, North-Holland, Amsterdam, 1982. MR745449 (85e:60001)

[6] T. W. Gamelin, Uniform algebras and Jensen measures, Cambridge University Press, London, 1978. MR521440 (81a:46058)

[7] P. Janakiraman, Best weak-type $(p, p)$ constants, $1 \leq p \leq 2$ for orthogonal harmonic functions and martingales, Illinois J. Math. 48, no. 3 (2004), 909-921. MR2114258 (2005m:60088)

[8] A. N. Kolmogorov, Sur les fonctions harmoniques conjugées et les séries de Fourier, Fund. Math. 7 (1925), 24-29.

[9] S. K. Pichorides, On the best values of the constants in the theorems of M. Riesz, Zygmund and Kolmogorov, Studia Math. 44 (1972), 165-179. MR0312140(47:702)

[10] M. Riesz, Sur les fonctions conjuguées, Math. Z. 27 (1928), 218-244. MR.1544909 
[11] G. Wang, Differential subordination and strong differential subordination for continuous-time martingales and related sharp inequalities, Ann. Probab. 23 (1995), 522-551. MR 1334160 (96b:60120)

[12] A. Zygmund, Trigonometric series, Vol. 2, Cambridge University Press, London, 1968. MR.0236587 (38:4882)

Department of Mathematics, Informatics and Mechanics, University of Warsaw, Banacha 2, 02-097 Warsaw, Poland

E-mail address: ados@mimuw.edu.pl 